\title{
BLOCK ADJUSTMENT OF LARGE-SCALE HIGH-RESOLUTION OPTICAL SATELLITE IMAGERY WITHOUT GCPS BASED ON THE GPU
}

\author{
Q. Fu ${ }^{1,2,3}$, S. Liu ${ }^{1,2, *}$, X. Tong ${ }^{1,2}$, H. Wang ${ }^{1,2}$ \\ ${ }^{1}$ College of surveying and Geo-informatics, Tongji University, Shanghai 200092, China \\ - (fuqing, liusjtj, xhtong, 1833546)@tongji.edu.cn \\ ${ }^{2}$ State Key Laboratory of Disaster Reduction in Civil Engineering, Tongji University, Shanghai 200092, China \\ ${ }^{3}$ School of electronics and information engineering, Jinggangshan University, Jiangxi Ji'an 343009, China
}

ICWG II/III: Pattern Analysis in Remote Sensing

KEY WORDS: Block adjustment, optical satellite imagery, large-scale, without GCPs, GPU

\begin{abstract}
:
Precise geo-positioning of high-resolution optical satellite imagery without ground control points (GCPs) has always been the goal pursued by photogrammetry scholars. This paper introduces the block adjustment (BA) without GCPs based on rational function model (RFM) model and its practical application in high-precision geo-positioning of optical satellite imagery. The mainly key technologies of BA model construction based on virtual control points (VCPs), gross error detection and elimination, and GPU parallel computing method of large-scale adjustment are studied. On this basis, experimental analysis and validation of 123 images of ZY-3 satellite in Taihu are carried out. The results show that the sparse matrix compression can reduce the memory requirement effectively. The GPU parallel computing can solve the problem of large-scale BA computational efficiency. In addition, after BA, the maximum residual is 3.79 pixel, the root mean square error (RMSE) is 0.37 pixel in the $x$ (flight) direction, the maximum residual error is 7.18 pixel, and the RMSE is 0.66 pixel in the $y$ (scan) direction. The proposed method has certain accuracy and stability in large-scale BA without GCPs. The relative positioning accuracy can reach sub-pixel level, which could meet the requirements of cartographic mosaicking.
\end{abstract}

\section{INTRODUCTION}

Under the background of global mapping, regions with little or no control point information, such as the plateau, alpine and gorge, desert and other areas without available ground control points (GCPs), are often encountered (Wang et al., 2017). Therefore, the block adjustment (BA) without GCPs of highresolution optical satellite imagery has attracted extensive attention of photogrammetricians. Traditional BA of optical satellite imagery requires a certain number of uniformly distributed GCPs. However, due to the influence of time, cost and policy, high-precision GCPs are usually difficult to obtain in practical application. Reducing the dependence on GCPs is to reduce cost and improve efficiency, which is efficient and effective method, and no GCPs and large-scale are the development trend of optical satellite imagery adjustment technology in the future (Wang et al., 2017).

Abroad common high-resolution optical satellite imagery, such as SPOT-5, IKONOS and QuickBird, does not have high accuracy of direct positioning. The original Rational Polynomial Coefficients (RPC) parameters contain obvious systematic error, so it is necessary to establish a corresponding error compensation model to compensate it (Zheng et al., 2016, Cheng et al., 2015, Tong et al., 2015, Ye et al., 2015 ). Grodecki et al. (2003) analysed the systematic error of RPC model systematically, and used affine transformation model to correct the systematic error of RPC model effectively. Zhang et al. (2016) improved the planar accuracy of SPOT-5 images from $13.7 \mathrm{~m}$ to $5 \mathrm{~m}$ after BA, and elevation accuracy from $9 \mathrm{~m}$ to less than $5 \mathrm{~m}$. In the case of sparse GCPs, BA experiments were carried out by using the constraint between different strip images, the influence of systematic error of RPC model was corrected effectively ( $\mathrm{Li}$ et al., 2006). Yao et al. (2018) adopted the BA method based on satellite images covered by multipletimes in the same place, which improved the accuracy of geopositioning of satellite images without GCPs. Li et al. (2018) used high-resolution satellite imagery with higher positioning accuracy (such as Geoeye, WorldView) or aerial images as control condition, and achieved better geo-positioning results. In addition, existing geographic information data, such as Digital Orthophoto Model (DOM) and Digital Elevation Model (DEM), could also be used as control data for combined BA of satellite imagery (Zhang et al., 2017). Zhou et al. (2016) used SRTM data to interpolate the elevation value of object points corresponding to tie points as the initial adjustment value, which improved the image elevation positioning accuracy of large-scale BA effectively. The geo-positioning accuracy of the BA of 8802 ZY-3 three-line array stereo images without GCPs covering the scope of China was better than $5 \mathrm{~m}$ (Wang et al., 2017).

In order to evaluate the ability of large-scale uncontrolled BA for the three-line array image of ZY-3 satellite, experimental analysis and validation of 123 images of ZY-3 satellite in Taihu are carried out. The results show that the maximum residual of the adjustment results is 3.79 pixel, the RMSE is 0.37 pixel in the $\mathrm{x}$ (flight) direction, the maximum residual is 7.18 pixel, and the RMSE value is 0.66 pixel in the y (scan) direction after BA. The proposed method has certain accuracy and stability in

\footnotetext{
* Corresponding author
} 
large-scale BA without GCPs. The geometric accuracy of the image is uniform, and the relative positioning accuracy can reach sub-pixel level, which could meet the requirements of cartographic mosaicking.

\section{METHODOLOGY}

\subsection{RFM model}

RFM model represents image-space coordinates $(r, c)$ as the ratio of polynomials whose independent variables are objectspace geodetic coordinates $(P, L, H)$, which essence is high mathematical fitting of the rigorous sensor model (RSM). It can achieve almost the same geometric positioning accuracy as the RSM. The basic form is shown in equation (1).

$$
\left\{\begin{array}{l}
r_{n}=\frac{\operatorname{Num}_{L}\left(P_{n}, L_{n}, H_{n}\right)}{\operatorname{Den}_{L}\left(P_{n}, L_{n}, H_{n}\right)} \\
c_{n}=\frac{\operatorname{Num}_{s}\left(P_{n}, L_{n}, H_{n}\right)}{\operatorname{Den}_{s}\left(P_{n}, L_{n}, H_{n}\right)}
\end{array}\right.
$$

Where $\left(r_{n}, c_{n}\right)\left(P_{n}, L_{n}, H_{n}\right)$ are the image-space coordinates and object-space coordinates after normalised respectively, and their values are between -1 and +1 .

The systematic error of the BA model is corrected by the additional image-space compensation model. Equation (1) can be rewritten as:

$$
\left\{\begin{array}{l}
r+\Delta r=F_{r}(P, L, H) \\
c+\Delta c=F_{c}(P, L, H)
\end{array}\right.
$$

Where $\left\{\begin{array}{l}\Delta r=a_{0}+a_{1} r+a_{2} c \\ \Delta c=b_{0}+b_{1} r+b_{2} c\end{array}\right.$ are the systematic error correction parameters in the line and sample direction of optical satellite imagery respectively, and $\left(a_{0}, a_{1}, a_{2}, b_{0}, b_{1}, b_{2}\right)$ are the compensation parameters of the systematic error. Equation (2) can be written as follows:

$$
\left\{\begin{array}{l}
G_{r}=r+\Delta r-F_{r}(P, L, H) \\
G_{c}=c+\Delta c-F_{c}(P, L, H)
\end{array}\right.
$$

$$
\text { Where }\left\{\begin{array}{l}
F_{r}(P, L, H)=\frac{\operatorname{Num}_{L}(P, L, H)}{\operatorname{Den}_{L}(P, L, H)} \cdot r_{s}+r_{0} . \\
F_{c}(P, L, H)=\frac{\operatorname{Num}_{s}(P, L, H)}{\operatorname{Den}_{s}(P, L, H)} \cdot c_{s}+c_{0}
\end{array}\right.
$$

After Equation (3) is linearized, the error equation of BA can be derived as:

$$
V=A t+B x-L, P
$$

Where $\boldsymbol{V}$ are the residual error vectors of the tie-point measurement in the line and sample directions, $\boldsymbol{A}, \boldsymbol{B}$ are the corresponding coefficient matrices respectively, $\boldsymbol{x}$ are the correction vectors for object-space geodetic coordinates corresponding to the tie points, $\boldsymbol{t}$ are the correction vectors for systematic error compensation parameters of image-space coordinates, $\boldsymbol{L}$ are constants calculated from the initial value, $\boldsymbol{P}$ are the corresponding weight matrices.

The Virtual Control Points (Yang et al., 2017) are obtained from forward intersection method of the object-space at any elevation datum level (generally set as the elevation offset in the initial RPC model of the image), which using the initial RPC model of the original image at the center of each grid. The regular grid is divided uniformly according to a certain distance in image-space ( 9 grids per image) for every image.

The solution of BA is an iterative procedure. When the results of two adjustment solution are less than the threshold value, the iterative procedure will be stopped.

\subsection{Sparse matrix compression}

In the procedure of large-scale BA, due to the large number of images involved, the number of unknown parameters in the normal equation is still very large. When the number of images in the BA is very large, the coefficient matrix of the normal equation is ill-conditioned. In addition, the memory requirement and the amount of computation caused by a large number of unknown parameters pose a great challenge to the general computer.

Considering that the coefficient matrix of the modified normal equation in the BA model has obvious sparsity, the data storage structure of sparse matrix is adopted in this paper $(\mathrm{Wu}$ et al., 2004). The data storage structure uses three metadata structure to record and store non-zero elements in each row of the sparse matrix. Each row contains the number of non-zero elements in the row, the column coordinates of each non-zero element and its corresponding values. With this data structure, only the nonzero elements in the matrix can be stored and calculated, thus avoiding arbitrary operation on the zero elements completely.

\subsection{Gross error detection and elimination}

Large-scale BA involves image matching between a large number of images. Without tie points selection strategy, the number of tie points obtained by automatic matching method is very large, and a large number of tie points have obvious gross error. When BA without GCPs, there are many gross error points which will directly lead to distortion of the regional network, and affect the accuracy and reliability of the adjustment results. In order to solve this problem, this paper adopts multi-stage gross error detection and elimination method (Wang et al., 2017), which reduces the influence of gross error on adjustment results effectively.

\subsection{GPU parallel computing}

At present, matrix inversion method mainly include QR decomposition, Jacobi, LU decomposition and minimal polynomial inversion method. When inverting very large-scale real symmetric matrix, these methods calculate slowly and can not meet the requirement of timeliness in practical engineering (Huo et al., 2015). To design parallel inversion method for very large-scale matrix, it is of great practical significance to improve the calculating efficiency on the premise of satisfying the calculation accuracy. In this paper, the modified normal equation in RFM model is a real symmetric matrix, which can be inverted by block iteration method.

The block iteration method of coefficient matrix of modified normal equation belongs to the calculation procedure of cyclic iteration, but each cycle procedure contains a large number of matrix multiplication and addition operations, and is independent of each other. Therefore, it can be optimized and computed in the GPU device. 


\section{EXPERIMENTS AND ANALYSIS}

\subsection{Experimental data}

In this paper, the BA experiment is carried out by using 123 images of ZY-3 three-line array stereo imagery in Taihu. The ZY-3 satellite imagery in this area is captured between 2015 and 2016. Each image is attached with original RPC parameter file. There exists a certain overlap between adjacent images. The total image data amount is about $190 \mathrm{~GB}$. The survey area covers an area of about $300 \mathrm{~km} \times 400 \mathrm{~km}$, which includes mountain, hill, plain and other topography, with the maximum and minimum elevation difference of $1500 \mathrm{~m}$.

\subsection{Experimental Solution}

The software environment used in the experiment is Windows 10 64-bit operating system, MATLAB R2017a professional numerical calculation software and DELL high performance graphics workstation (the graphics workstation is equipped with a 12-core i7-8700 CPU, 32GB memory and NVIDIA Quadro P2000 professional graphics card). The LPS module of ERDAS IMAGINE 2015 automatically matches about 37204 uniform tie points in the whole measuring area, which costs about 4 hours. The each image is uniformly divided into $3 \times 3$ grid in imagespace. VCPs are generated at the center of each grid, and 9 VCPs are generated on each image. A total of 1107 VCPs are generated. Combined BA experiments are carried out between the generated VCPs and the matched tie points. The experimental results show that introducing VCPs into the traditional free network adjustment model as weighted observations can effectively improve the condition of the adjustment model and make the adjustment model have better robustness.

\subsection{Results and analysis}

The proposed method requests much less memory space than the traditional method. When the number of images reaches 123 in the experiment, the traditional method needs at least $80 \mathrm{~GB}$ memory space to carry out adjustment calculation. However, the proposed method only needs about $2178 \mathrm{MB}$ memory space (among which $1216 \mathrm{MB}$ is occupied by the free state of MATLAB software), which occupies less memory space. The traditional method fails in memory allocation and could not carry out adjustment calculation. When preconditioned conjugate gradient (PCG) method (Zheng et al., 2017) is used for adjustment, the adjustment costs 150.722 second. When GPU device is used for parallel calculation, the adjustment costs 49.327 second. When combined block matrix and GPU parallel calculation technology are used for calculation operation, the adjustment costs the least time, only 28.945 second.

Analysis of the re-projection errors of the tie points after BA and comparison with the re-projection errors of the tie points after directly forward intersection which using the original RPC parameter can evaluate the accuracy and stability of the BA, which can also reflect the relative positioning accuracy of the images to some extent. The relative positioning accuracy before and after adjustment is shown in Figure 1. After BA, the maximum residual is 3.79 pixel, the RMSE is 0.37 pixel in the $x$ (flight) direction, the maximum residual is 7.18 pixel, and the RMSE is 0.66 pixel in the $y$ (scan) direction .

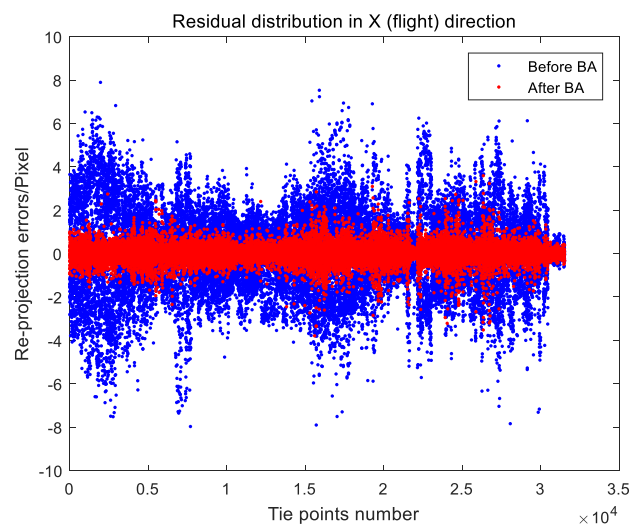

(a) Re-projection error in $x$ direction

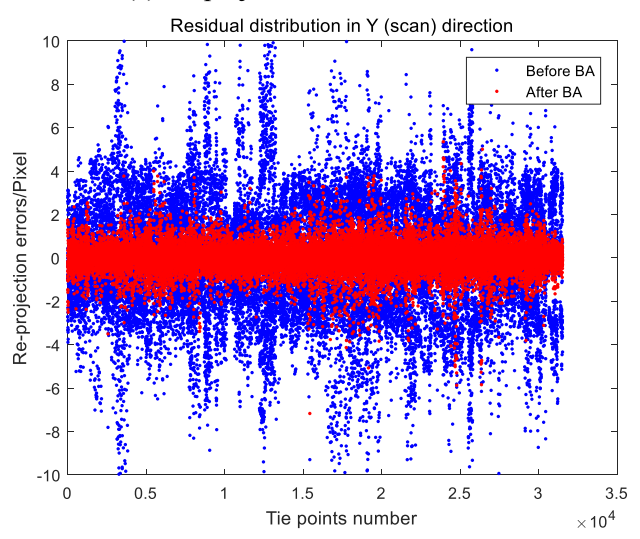

(b) Re-projection error in $y$ direction

Figure 1. Re-projection error of tie points.

\section{CONCLUSIONS}

In this paper, based on RFM model, the constraints between high-resolution optical satellite imagery are utilized to compensate the systematic errors of ZY-3 satellite by establishing affine transformation in image-space. Aiming at the memory requirement and the efficiency of adjustment solution of large-scale BA method, sparse matrix storage compression, robust and efficient adjustment solution method are adopted. Making full use of the advantages of sparse matrix, the memory requirement is reduced. After modifying the normal equation, the GPU parallel computing method and block matrix iteration optimization operation are carried out.

The BA without GCPs is carried out by using 123 images of ZY-3 three-line array satellite imagery in Taihu. The experimental results show that the BA model constructed by the constraints relationship between images can compensate the systematic error of RPC model well. After introducing VCPs, the ill-conditioned problem of modified normal equation in the adjustment procedure can be solved effectively, which makes the adjustment result better robustness. After BA, the maximum residual is 3.79 pixel, the RMSE is 0.37 pixel in $x$ (flight) direction and the maximum residual is 7.18 pixel, the RMSE is 0.66 pixel in $y$ (scan) direction. This shows that the proposed method has certain accuracy and stability in large-scale BA without GCPs. The relative positioning accuracy can reach subpixel level, which could meet the requirements of cartographic mosaicking. Due to the limitation of experimental data, the correctness and feasibility of the proposed method still need to be further validated by a large number of different types of 
optical satellite imagery, and how to make full use of the existing geographic information data to assist the adjustment of large-scale BA, which will be the next research direction of this paper.

\section{ACKNOWLEDGEMENTS}

The work described in this paper was jointly supported by the National Key R\&D Program of China (Project no. 2017YFB0502705), the National Natural Science Foundation of China (Project no. 41771483), the Research Fund from the State Key Laboratory for Disaster Reduction in Civil Engineering(Project no. SLDRCE19-B-36), Jiangxi Education Department Science and Technology Project (Project no. GJJ170651), Natural Science Foundation of Jinggangshan University (Project no. JZ1703), and the Fundamental Research Funds for the Central Universities.

\section{REFERENCES}

Cheng, C., Zhang, J., Huang, G., 2015. RFM-Based Block Adjustment for Spaceborne Images with Weak Convergent Geometry. ISPRS - International Archives of the Photogrammetry, Remote Sensing and Spatial Information Sciences, XL-7(W4), 1-6.

Grodecki, J., Dial, G., 2003. Block Adjustment of HighResolution Satellite Images Described by Rational Polynomials. Photogrammetric Engineering \& Remote Sensing, 69(1), 59-70.

Huo, Y., Wang, W., Peng, C., Fang Y., 2015. CUDA-based parallel matrix inversion algorithm of large-scale real symmetric matrix. Computer Engineering and Design, (8), 2133-2137.

Li, H., Cao, H., Shi, J., 2018. High-precision Orientation Algorithms of High-resolution Satellite Imagery. Geospatial information, 16(105), 11-18.

Tong, X., Xu, Y., Ye, Z., Liu, S., Tang, X., Li, L., Xie, H., Xie, J., 2015. Attitude Oscillation Detection of the ZY-3 Satellite by Using Multispectral Parallax Images. IEEE Transactions on Geoscience and Remote Sensing, 53(6), 3522-3534.

Wang, M., Yang, B., Li, D., Gong, J., Pi, Y., 2017. Technologies and Applications of Block Adjustment Without Control for ZY-3 Images Covering China. Geomatics and Information Science of Wuhan University, 42(4), 427-433.

Wu, J., Wang, Z., Li, X., 2004. Efficient Solution and Parallel Computing of Sparse Linear Equations. Hunan Science and Technology Press.

Yang, B., Wang, M., Pi, Y., 2017. Block-adjustment without GCPs for Large-scale Regions only Based on the Virtual Control Points. Acta Geodaetica et Cartographica Sinica, 46(7), 874-881.

Yao, X., You, H., 2018. Multi-Observed Block Adjustment for Satellite Images Without Ground Control Points. Remote Sensing Technology and Application, 33(3), 555-562.

Ye, Z., Xu, Y., Wang, F., Liu, S., Tang, X., Li, L., Xie, J., Tong, X., 2015. Estimation of the Attitude Perturbance Using Parallax Imagery-Application to ZY-3 Satellite. ISPRS Annals of the
Photogrammetry, Remote Sensing and Spatial Information Sciences, 1, 279-283.

Zhang, Y., Wan, Y., Huang, X., Ling, X., 2016. DEM-Assisted RFM Block Adjustment of Pushbroom Nadir Viewing HRS Imagery. IEEE Transactions on Geoscience and Remote Sensing, 54(2), 1025-1034.

Zhang, Z., Tao, P., 2017. An Overview on "Cloud Control" Photogrammetry in Big Data Era. Acta Geodaetica et Cartographica Sinica, 46(10), 1238-1248.

Zheng, M., Zhang, Y., Zhou, S., Zhu, J., Xiong, X., 2016. Bundle block adjustment of large-scale remote sensing data with Block-based Sparse Matrix Compression combined with Preconditioned Conjugate Gradient. Computers \& Geosciences, 92, 70-78.

Zhou, P., Tang, X., Cao, N., Wang, X., Li, G., Zhang, H., 2016. SRTM-aided Stereo Image Block Adjustment without Ground Control Points. Acta Geodaetica et Cartographica Sinica, 45(11), 1318-1327. 